\title{
Performance comparison of queuing algorithms: a review
}

\author{
Kamalpreet Kaur ${ }^{1}$, Navdeep Kaur ${ }^{2}$ Gurjeevan Singh ${ }^{3}$ \\ ${ }^{I}$ (M.Tech Scholar, SBSSTC, FZR, INDIA) \\ ${ }^{2}(A P, E C E$ deptt, SBSSTC, FZR, INDIA) \\ ${ }^{3}(D I C-E C E, S B S S T C$ (P.W.), FZR, INDIA)
}

\begin{abstract}
Denial of service attacks produce very large amount of packets by a large amount of agents, these packets can easily interfere with the original flow and communication source within very short duration of time. The low rate distributed denial of service attacks (LDDoS) is those which produce less amounts of packet to attack flows. They are a combination of a large number of $L D o S$ flows. There are several techniques called queuing algorithms which are introduced to fight $L D D o S$ attacks. In our research work we will compare some of these queuing management techniques to find the best among them. This review paper will be helpful in our further work.
\end{abstract}

Keywords: BLUE, CBQ, DROPTAIL, RED, SFQ

\section{INTRODUCTION}

Today's internet services face the major threat in form of denial of service attacks. TCP-targeted low rate distributed denial of service attacks are the new type of attacks which are more efficient in terms of causing damage to legitimate flows and moreover are very difficult to detect when compared to traditional flooding based DDoS attacks. [1]

NS is computer network software and an event-based simulator being designed and implemented in California (Berkley) University. It can simulate a wide spectrum of the protocols. The locations in which the data packages are kept or dropped forms the queues. In queuing, a plan of the packages is needed, there should be a process on which a decision that which packet is to be kept and which is to be dropped. This process is called as "buffer management". The thing which is important in queue disciplines is the volume of the packages which has been kept and dropped and calculation of the throughput of the network. The issues related to the efficiency are of great importance. When hundreds and thousands computers are linked to each other, their multifaceted communication is with unexpected outcomes and most of the time, these complexities lead into the weak efficiency of the network and nobody knows what the reason is. Some of the efficiency problems are created due to excess use of the present resources. If suddenly there is excess traffic through the router, congestion is created.

Queuing algorithms which are implemented in NS2 software and compared are droptail, RED, BLUE, SFQ, SRR and CBQ.

\subsection{QUEUE MANAGEMENT ALGORITHMS}

The queuing algorithms which have been used are discussed briefly here.

\subsubsection{DROPTAIL}

Drop Tail is a simple queue management algorithm: it sets a predefined value for the maximum length of the queue and when this value is reached, new packets are discarded, until the next vacant buffer space to accept new packets. When using the Drop Tail mechanism, all the packets in the traffic are treated identically, regardless of the type of traffic which it belongs to. Packet loss will cause the transmitter to reduce the number of TCP packets sent before receiving the acknowledgment. The throughput of the a given TCP session will then reduce, until the transmitter start again to receive acknowledgments and begin increasing the size of its congestion window. [3]

\subsubsection{RED}

RED (Random Early Detection) works by randomly (based on certain probability) discarding packets at the nodes of the network, before the occurrence of congestion, when the average queue length exceeds the predefined minimum threshold. When the average queue length exceeds the maximum threshold, the probability of rejection becomes equal to 1 . RED monitors the average length of the queues by discarding or ECN-marking packets based on statistical probability. If the buffer is nearly vacant, all incoming packets are received. As there is increase in use, the probability of discarding recently arrived packet also increases. When the buffer is 
occupied, all incoming packets are deleted. RED has no QoS differentiation in the basic version. The versions WRED (Weighted RED) and RIO (RED with In and Out), which consider the QoS into account. [3]

\subsubsection{BLUE}

BLUE algorithm uses packet loss and record of use to deal with the link queue. During that operation, it accidentally deletes or ECN-marks packets in the queue on the router before the queue become occupied. BLUE maintains the probability of deleting/marking $\mathrm{p}$, and on the basis of these probabilities, it deletes or marks packets when they come into the queue. When the queue is occupied, $p$ increases by a constant value of $\mathrm{pd}$, and when the queue is vacant, $\mathrm{p}$ is reduced to a constant value of $\mathrm{pi}<\mathrm{pd}$. If the packet is deleted due to the buffer possession, BLUE increases the value of $\mathrm{p}$ by increasing the rate at which congestion notification is sent back or those packets are deleted. The main drawback is that the BLUE algorithm has no dissimilar flow rates, but treats all flows together as collective. Therefore, it may happen that a forceful flow ejects the packets from other, less forceful flow. [3]

\subsubsection{CLASS BASED QUEUING (CBQ)}

This algorithm is also called Weighted Round Robin (WRR); the packets are divided into various service classes and are then transferred into the queue that is well thought-out for the service and each of the queues are serviced in a quantum.[3] Different bandwidth at the output port to each queue can be assigned.

\subsubsection{STOCHASTIC FAIR QUEUING (SFQ)}

It is modified type of FQ by the aim of removing its limitations as this method reduces the number of required queues. One of the most important drawbacks of this method is unfair behavior with the flows colliding with other flows. Thus, as the name reveals, fair is guaranteed as stochastically. [2]

It is suitable for use in high speed computer networks that covers a wide range of CPU, memory and fairness trade-offs. It offers elegant degradation under overload and sudden failure. [6]

\subsubsection{SRR}

SRR is a shaping mechanism that limits egress traffic on a per-queue basis. SRR is configured by assigning a maximum weight to each queue, which is converted to a percentage of link bandwidth. The traffic in each queue is then shaped to that percentage. SRR only allows a queue to use the specific amount of bandwidth that the weight allocates. If you do not enable SRR, weighted round robin (WRR) is used. [9]

The Cisco Catalyst 3560-E employs Shaped Round Robin (SRR). SRR is scheduling service for specifying the rate at which packets are dequeued. With SRR there are two modes, shaped and shared. Shaped mode is only available on the egress queues. Shaped egress queues reserve a set of port bandwidth and then send evenly spaced packets as per the reservation. Shared egress queues are also guaranteed a configured share of bandwidth, but do not reserve the bandwidth. That is, in shared mode, if a higher priority queue is empty, instead of the servicer waiting for that reserved bandwidth to expire, the lower priority queue can take the unused bandwidth. Neither shaped SRR nor shared SRR is better than the other. Shared SRR is used to get the maximum efficiency out of a queuing system, because unused time slots can be reused by queues with excess traffic. This is not possible in a standard weighted reservation. Shared egress queues are also guaranteed a configured share of bandwidth, but do not reserve the bandwidth. That is, in shared mode, if a higher priority queue is empty, instead of the servicer waiting for that reserved bandwidth to expire, the lower priority queue can take the unused bandwidth. Neither shaped SRR nor shared SRR is better than the other. Shared SRR is used to get the maximum efficiency out of a queuing system, because unused time slots can be reused by queues with excess traffic. This is not possible in a standard Weighted Round Robin (WRR). Shaped SRR is used to shape a queue or set a hard limit on how much bandwidth a queue can use. When you use shaped SRR, you can shape queues within a port's overall shaped rate. [10]

\begin{tabular}{|c|c|c|c|c|}
\hline $\begin{array}{l}\text { Paper and year of } \\
\text { publication }\end{array}$ & Queuing algorithms used & $\begin{array}{c}\text { Traffic } \\
\text { patterns }\end{array}$ & Parameters & Conclusion \\
\hline $\begin{array}{l}\text { Flow level } \\
\text { detection, Year-2012 }\end{array}$ & RED \& Droptail & TCP & Packet drop & $\begin{array}{l}\text { RED is better than Droptail and DFT in } \\
\text { dropping more number of packets. }\end{array}$ \\
\hline $\begin{array}{ll}\text { The evaluation } & \text { of } \\
\text { behaviour } & \text { of } \\
\text { computer } & \\
\text { networks }(2013) & \end{array}$ & $\begin{array}{l}\text { CBQ, SFQ, DRR, FQ, } \\
\text { RED, Droptail }\end{array}$ & $\begin{array}{l}\text { TCP } \\
\text { UDP }\end{array}$ & Packet drop & $\begin{array}{l}\text { FQ and SFQ are best as they deliver maximum } \\
\text { number of packets, there is no change in case } \\
\text { of DRR, Droptail and RED drop max. Packets }\end{array}$ \\
\hline $\begin{array}{l}\text { Queuing algorithm } \\
\text { performance(2011) }\end{array}$ & $\begin{array}{l}\text { Droptail, } \\
\text { SFQ,DRR,RED }\end{array}$ & $\begin{array}{l}\text { TCP } \\
\text { UDP }\end{array}$ & $\begin{array}{l}\text { Buffer size } \\
\text { Attack } \\
\text { intensities }\end{array}$ & $\begin{array}{l}\text { Droptail and RED gives best performance for } \\
\text { buffer size } 60 \text {. FQ, SFQ and DRR are not } \\
\text { affected. SFQ is best against attack intensities } \\
\text { for buffer size } 60 \text {. }\end{array}$ \\
\hline $\begin{array}{l}\text { Performance } \\
\text { analysis and QOS }\end{array}$ & $\begin{array}{l}\text { Droptail, RED,SFQ, FQ, } \\
\text { DRR }\end{array}$ & $\begin{array}{l}\text { CBR } \\
\text { FTP }\end{array}$ & $\begin{array}{l}\text { Throughput } \\
\text { End-to-end }\end{array}$ & $\begin{array}{l}\text { FQ gives maximum throughput and RED is } \\
\text { worst. FQ gives maximum delay, RED gives }\end{array}$ \\
\hline
\end{tabular}




\begin{tabular}{|l|l|l|l|}
\hline & Pelay & $\begin{array}{l}\text { minimum delay. SFQ gives maximum packet } \\
\text { loss for CBR.RED gives maximum packet loss } \\
\text { for FTP, FQ gives minimum packet loss for } \\
\text { both CBR and FTP. }\end{array}$ \\
\hline
\end{tabular}

Table 1: Performance characteristics of the given algorithms

\section{CONCLUSION}

RED gives best performance at buffer size 60 and drops the maximum attack packets and gives minimum delay. SFQ delivers maximum number of packets and is best against attack intensities at buffer size 60. FQ gives maximum throughput and minimum packet loss. So from all the algorithms studied in our literature work, these three queuing algorithms give the best performance for different scenarios. We will compare the six algorithms BLUE, CBQ, DROPTAIL, RED, and SFQ to find out which one of them gives the best performance.

\section{REFERENCES}

[1] Changwang Zhang, Zhiping Cai, Weifeng Chen, Xiapu Luo, Jianping Yin, Flow level detection and filtering of low-rate DDoS attacks, SciVerse science direct, Computer Networks 56 (2012) 3417-3431

[2] Saman Afrasiabi, Farzaneh Abazari, The evaluation of the behavior of computer networks by NS simulator and the effect of queuing systems in the performance of especial networks, Life Science Journal 2013;10(1)

[3] Mohit Agrawal1, Navneet Tiwari, Lalla Atul Singh Chaurasia and Jatan Saraf, Performance Comparison of Active Queue Management Algorithms, 2009 International Symposium on Computing, Communication, and Control (ISCCC 2009) Proc .of CSIT vol.1 (2011) () (2011) IACSIT Press, Singapore

[4] E McKenney, ibm Beaverton, Stochastic Fair Queuing paul ,supported by Rome Air Development center and the Defense Advanced Research Projects Agency under contract number F30602-89-C-0015, and SRI internal Research and Development.

[5] Shaveta, Harsh Verma, Ashish kumar, Performance Evaluation of AQM Algorithms for PGM based group communication in PIMDM Multicasting Network, International Journal of Computer Science \& Engineering Technology (IJCSET), Vol. 4 No. 06 Jun 2013.

[6] Ashish Kumar, Ajay K Sharma, Arun Singh, Comparison and Analysis of Drop Tail and RED Queuing Methodology in PIM-DM Multicasting Network, (IJCSIT) International Journal of Computer Science and Information Technologies, Vol. 3 (2) , 2012,3816 3820

[7] Serhat ÖZEKES, evaluation of active queue management algorithms, Istanbul Ticaret Üniversitesi Fen Bilimleri Dergisi Yll:4 Sayl: 7 Bahar 2005/1 s.123-140

[8] Bin Xiao - Wei Chen · Yanxiang He, A novel approach to detecting DDoS attacks At an early stage, J Supercomput (2006) 36:235248 DOI 10.1007/s11227-006-8295-0

[9] (http://tools.cisco.com/ITDIT/CFN/Dispatch?act=featdesc\&task=display\&featureId=6502)

[10] (http://www.cisco.com/en/US/prod/collateral/switches/ps5718/ps7078/prod_qas0900aecd805bacc7.html) 